\title{
ToAM: A Task-oriented Authentication Model for UAVs Based on Blockchain
}

\author{
Aiguo Chen \\ University of Electronic Science and Technology of China \\ Kun Peng \\ University of Electronic Science and Technology of China \\ Zexin Sha \\ University of Electronic Science and Technology of China \\ Zhen Yang \\ Information Center of China North Industries Group \\ Guoming Lu ( $\nabla$ lugm@uestc.edu.cn )
}

University of Electronic Science and Technology of China https://orcid.org/0000-0001-7477-5800

\section{Research}

Keywords: Authentication, blockchain, unmanned aerial vehicles, smart contract

Posted Date: October 14th, 2020

DOI: https://doi.org/10.21203/rs.3.rs-90900/v1

License: (c) (i) This work is licensed under a Creative Commons Attribution 4.0 International License. Read Full License

Version of Record: A version of this preprint was published at EURASIP Journal on Wireless Communications and Networking on August 14th, 2021. See the published version at https://doi.org/10.1186/s13638-021-02039-6. 


\title{
ToAM: A Task-oriented Authentication Model for UAVs Based on Blockchain
}

\author{
Aiguo Chen ${ }^{1,2}$, Kun Peng ${ }^{1,2}$, Zexin Sha ${ }^{1,2}$, Zhen Yang ${ }^{3}$, Guoming Lu*1,2 \\ 1. School of Computer Science and Engineering, University of Electronic Science and Technology of China, \\ Chengdu 611731, China; \\ 2. Trusted Cloud Computing and Big Data Key Laboratory of Sichuan Province, Chengdu 611731, China; \\ 3. Information Center of China North Industries Group, Beijing 100089, China \\ $\underline{\text { *Correspondence: lugm } @ \text {,uestc.edu.cn }}$
}

\begin{abstract}
The pervasive collaboration of groups of UAVs has become vogue and popularized due to the reduced cost and widely adoption of these gadgets. It is believed that such collaboration will benefits numerous scenarios like the disaster monitoring and combat in battlefield. In these scenarios where UAVs collaborate to carry out tasks, the efficient and reliable authentication between UAVs is imperative. However, there are some major challenges hindering the authentication, including UAVs' resource limitation, joint using of cross-domain drones, and weakly connected task network environment. Therefore, a novel Task-oriented Authentication Model for UAVs Based on Blockchain (ToAM) is proposed. The framework takes advantage of the tamperresistant properties of the blockchain, and uses smart contract to achieve decentralized identity authentication and reduce the computing cost of UAVs. Analyzing results demonstrates that the authentication model can achieve lightweight, offline secure identity authentication for UAVs.
\end{abstract}

Keywords - Authentication; blockchain; unmanned aerial vehicles; smart contract

\section{Introduction}

Unmanned aerial vehicles (UAVs), also known as drones, are one type of aircrafts which operate without a human pilot onboard[1]. Due to its low-cost, low-risk and strong maneuverability, it is widely used in firefighting, search, rescue and so on[2]. In some dangerous environment, UAVs can be used to do some works which used to be done by humans, this can greatly reduce the incidence of security accident[3]. With the advancement of technology, the operation methods and usage scenarios of UAVs have also been greatly developed. Traditionally, the UAV carries out its mission independently under human control. Now, with the development of technology and the improvement of software and hardware, tasks could be completed by a group of UAVs[4][5]. Based on the information collected in real time, UAVs can communicate and collaborate within a group to complete tasks. And the whole process does not require human involvement.

To achieve the autonomous cooperation of UAVs mentioned above, the authentication between different UAVs must be solved firstly. However, there are many challenges in UAVs authentication, as is noted from the following aspects: 
- Resource Limitations. Due to resource limitations, UAVs cannot perform highintensity cryptographic computation. Therefore, the authentication protocols between UAVs need to be lightweight, as is expected for all power-constrained units [6].

- Unavailability of Centralized Control. Single point of failure issues may occur in a centralized authentication scheme. UAVs always need to go deep into outfield environment, in such an application scenario, a decentralized scheme is desperately needed.

- Cross-domain Issues. In a variety of typical scenarios, UAVs from different domains will collaborate in same task. Therefore, a model is needed to support effective mutual authentication between UAVs from different domains.

- Weak Connection. When UAVs cruise deeply into the conflict area with poor network connectivity, the existing connections within the UAVs will become extremely unstable. Therefore, the authentication scheme needs to support the mutual authentication within the UAVs when the network is unavailable.

Up to now, some work on UAVs authentication has been carried out, and a great deal of solutions have been proposed to these challenges. For example, In view of the resource limitation of UAV, various lightweight schemes have been proposed [7][8]. To achieve cross-domain access of UAVs, the authentication framework based on the symmetric key management and negotiation techniques has been designed [9]. Considering the high security and availability, the PKI technology has been widely used [10]. Meanwhile, new technologies like the blockchain (BC for short) keep emerging to benefit more reliable and flexible authentication [11]. For example, when dealing with the resource constrained environment, the BC technology converts the authentication from a heavy-calculation task to a problem can be solved by means of retrieval. It can tremendously reduce the complexity of authentication process and solve the difficulty of single point failure within PKI. Moreover, existing authentication schemes cannot rationally cope with the problem of weak connections faced by UAV groups

In this paper, to solve the challenges discussed above, we put forward a novel taskoriented Authentication Model for UAVs Based on Blockchain, which is a task-oriented and dynamic method, and could adapt better to the complex and changeable situation. Meanwhile, to reduce the computing cost of drones, this scheme uses the retrieval operation of blockchain to replace the complicated calculation, making the authentication process more simple and effective. Moreover, it is able to provide crossdomain access while resist against well-known attacks such as replay, key impersonation, and password guessing. Experimental results show that the authentication scheme ensures a stable relationship between the drones, even in a network-free environment. 
The rest of the paper is organized as follows. Section 2 reviews the existing related works about the application of blockchain and authentication mechanisms of UAV. Section 3 gives the overview and design details of our proposed solution. Section 4 discusses the security performance and evaluation about our authentication mechanism. Finally, Section 5 concludes the paper.

\section{Related Works}

In the area of UAVs authentication, several efforts have been made. In 2017, a data authentication scheme for UAVs ad hoc network communication was proposed[12], which uses the self characteristic of data to generate an authentication watermark and insert it into the data at random to ensure the security of data authentication process between drones, and the experimental results showed that their strategy can resist most attacks. In addition to the security problem, the lightweight of authentication is also a noteworthy issue. In [13], Chin-Ling Chen et al. proposed a traceable and privacypreserving authentication scheme for UAV, which uses a signature mechanism based on ECC (elliptic curve cryptography) to ensure data integrity and non-repudiation. Compared with the traditional signature mechanism, the application of ECC technology makes the authentication process more lightweight $[14,15]$.

Traditional UAV certification is mostly centralized and static. In [16], the registered flight session key is used to authenticate the drone by ground station. In order to achieve higher efficiency, Wang Hong et al. proposed a provably secure aggregate authentication scheme for UAVs cluster networks[17], their works secure that all kinds of data from different UAVs can be checked by batch instead of "one-by-one", and UAVs are allowed to join in dynamically at any moment.

Although these key establishment and privacy preserving schemes currently do exist for UAVs authentication, they are cannot fully handle all challenges mentioned in the first section. In terms of the computational cost of authentication, most of them rely on the high computational costs to ensure the security, and ignore the characteristic of low computing power of UAVs. In addition, these authentication schemes ignore the cross-domain applications of UAVs and have no corresponding design [18] for this part. What's more, the existing schemes are always static rather than task-oriented, which restrict the practical application of UAVs. Lastly, almost all of these schemes fail to consider the challenge of weak connection, when the UAVs is deep behind enemy or the network environment is not available, the authentication between UAVs becomes a problem.

Fortunately, blockchain has been proved to be used to improve the security, reduce the complexity of authentication, and achieve the effect of lightweight effectively[19]. Blockchain is a chain of blocks which is connected by saving the hash value of the 
previous block [20]. The block records the information of transactions, each transaction taking place on the $\mathrm{BC}$ needs the signature of the previous owner. In essence, $\mathrm{BC}$ is a distributed database. Anyone who wants to temper with the data in the blockchain must at least master $51 \%$ computing power of the $\mathrm{BC}$. Considering those characteristic, BC is seen as reliable, secure and the information stored on it is untamperable. Some researchers have made efforts on combining blockchain with practical applications.

In this paper, we creatively combine the UAVs authentication process with blockchain, and propose a dynamic and task-oriented authentication model, which also provides a guarantee for the cross-domain access of UAVs, while taking the problems caused by weak connection into account.

\section{Methods}

\subsection{System Model}

To solve the authentication challenges of UAVs, such as resource limitations, the pressure of centralization, cross-domain access and the weak connection mentioned above. A new type of Task-oriented Authentication Model for UAVs Based on Blockchain (ToAM) is proposed. In our model, all entities serve as nodes in the blockchain, and the entire system is structured into three layers and multiple domains. The system model is shown in Figure 1.

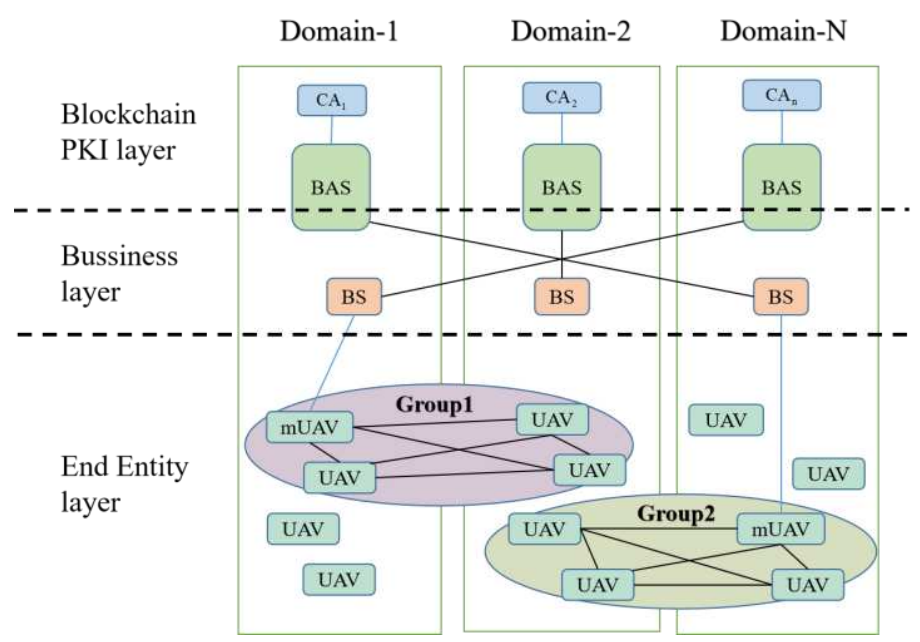

Figure 1 ToAM system model

In the Blockchain PKI layer, there is a CA(Certificate Authority) to manage the identity information of the devices in each domain. These top-level CAs jointly maintain a blockchain network for publishing, storing, and maintaining identity information.

The business processing layer contains BAS (Blockchain Authentication Server) and BS (Business Server).

- BAS: As an entity in the blockchain network, it plays the role of the master node. It can store the certificate information published by CA in the blockchain network, or assist drones in querying the certificate information of other entities. It can be 
understood as a blockchain agent of UAV.

- BS: As an entity in the blockchain network, it is responsible for publishing task information to UAV and can maintain task information in the blockchain.

Both of them have the blockchain network accessing function. Together with the master nodes in other domains, they build a blockchain network for publishing and storing information such as identities and tasks.

In the End Entity layer, there are multiple UAVs in each domain. When a UAV accepts a task, it will automatically be organized and scheduled according to the task goal, and cooperate with others to complete the task. We assume that the local storage of the UAV uses TPM(Trusted Platform Module), and the authentication information stored by the drone can not be read through violent destruction.

When BS publishes a task, it will filter out a UAV as master UAV (mUAV). This mUAV will work as the founder of a UAV Group in the future. After that, mUAV sends identity certificates to the appropriate UAVs according to the instructions (In fact, it can not be called an identity certificate. We call it Ticket). When the UAV confirms the authenticity of the mUAV identity, it will save the temporary identity created by the mUAV, and immediately call the smart contract to submit its encrypted temporary identity to the blockchain.

In the subsequent internal interaction process of the UAV group, the UAV will use its own temporary identity, which can be proved through the blockchain. The details are described from the subsection 3.3 to subsection 3.6.

\subsection{Presumption and Definition}

Mainly focusing on the progress of authentication of UAVs, we put forward following presumptions to reduce the interference caused by unrelated factors and build the basic functions that UAVs must have.

- All UAVs have been registered with CA in the domain, and have been registered in the blockchain network.

- The UAV that accepts the mission will strictly implement the mission requirements and will not generate false mission intelligence.

- We do not consider physical attacks, and UAVs use TPM(Trusted Platform Module) to ensure that important local data will not be violently read by attackers.

The symbols used in this paper are defined in the following Table 1.

Table 1 Definition of symbols

\begin{tabular}{|c|l|}
\hline Symbols & \multicolumn{1}{c|}{ Definition } \\
\hline GrpID & The group ID of a UAV group \\
\hline ID $_{x}$ & The temporary identity of drone x \\
\hline Ticket & Task tickets issued by mUAV for other UAVs \\
\hline
\end{tabular}




\begin{tabular}{|c|l|}
\hline $\mathrm{P}_{\mathrm{a}}, \mathrm{P}_{b}$ & UAV alb's public key \\
\hline $\mathrm{S}_{\mathrm{a}}, \mathrm{S}_{b}$ & UAV alb's private key \\
\hline $\boldsymbol{S} \boldsymbol{K}$ & Session key \\
\hline $\boldsymbol{E}_{\boldsymbol{K}}(\cdot)$ & Encryption using key K \\
\hline $\boldsymbol{H}(\cdot)$ & Hash function \\
\hline $\boldsymbol{S i g n}(\cdot)$ & signature \\
\hline $\boldsymbol{T S}$ & Timestamp \\
\hline
\end{tabular}

We use GrpID to identify the existence of a UAVs group, and make it public to the outside world to make others perceive the existence of drone groups. In addition, we assigned a unique $\mathrm{ID}_{x}$ to each UAV in the group, And the value is public within the group, We assume that any UAV that has joined the group will not actively disclose $\mathrm{ID}_{x}$ outside the group.

$\mathrm{E}_{\mathrm{K}}(\cdot)$ represents an encryption operation. Specific algorithms can be flexibly selected here according to actual business scenarios.

In addition, since the hash function and signature function involve the operation of blockchain smart contracts, it is necessary to use the blockchain signature function ECDHE and the blockchain standard hash function Keccak3. The cross-domain authentication mechanism exploits ECDHE, which is based on the elliptic curve discrete logarithm problem (ECDLP). ECDLP has been proved unsolvable by polynomial-time algorithm now. In addition, all the information required identity authentication, such as the public key of UAV, is stored on the blockchain, which connects all domains and provides tamper-resistant record service. Besides, the privacy security of UAV's identity and data integrity of transactions and messages is more depending on the hash function. The Keccak3 hash function has been used in this paper, which has never been compromised yet.

\subsection{Group Initialization}

In this phrase, the mUAV that receives the task from BS will distribute tickets to the appropriate UAVs. We assumed that the mUAV here must be honest and reliable. 
The specific process is shown in Figure 2.

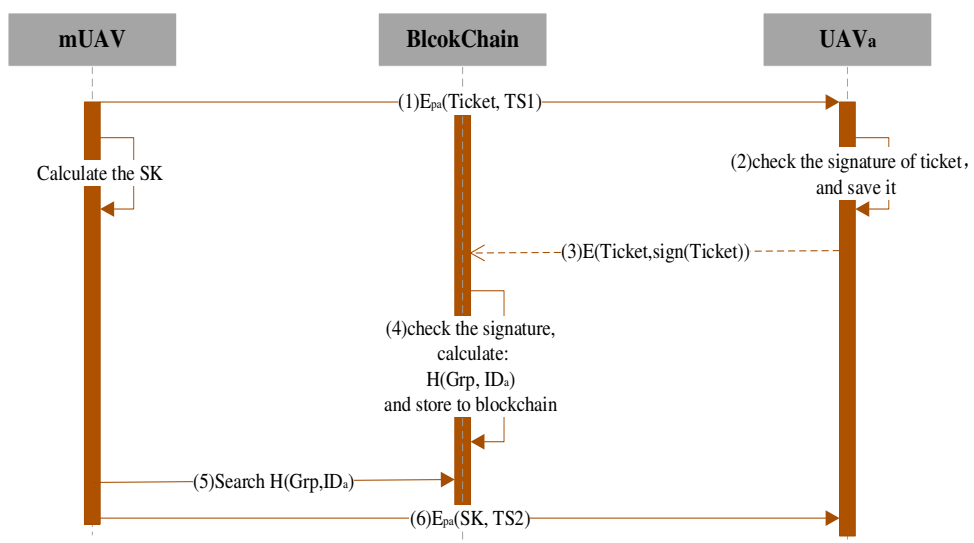

Figure 2 Initialization

The protocol steps are as follows:

Step (0): When mUAV receives a task, it generates a Ticket for each UAV selected as follows:

Ticket_to_A $=\left\{G r p I D\left|I D_{A}\right| \operatorname{sign}\left(G r p I D, I D_{A}\right)\right\}$

where, GrpID represents the unique identifier of the UAV group that established by mUAV, which is public. $\mathrm{ID}_{A}$ is a private identification generated by mUAV for $\mathrm{UAV}_{A}$. Sign $\left(\mathrm{GrpID}, \mathrm{ID}_{A}\right)$ is a signature, which is signed by mUAV's private key.

Step (1): After finding the public key of $\mathrm{UAV}_{a}$ through the blockchain, mUAV uses this public key to encrypt and sends $\left\{\right.$ Ticket, TS1\} to $\mathrm{UAV}_{a}$.

$$
\text { mUAV } \stackrel{E_{\mathrm{pa}\{\text { Ticket, } T S 1\}}}{\longrightarrow} U A V_{a}
$$

Step (2): After $U_{A} V_{a}$ decrypts message with its private key, it judges the timestamp TS1, then obtains the public key of mUAV through the blockchain, checks the signature in the ticket, and after confirming the authenticity of the ticket, saves GrpID and $\mathrm{ID}_{a}$.

Steps (3)-(4): $\mathrm{UAV}_{a}$ calls the smart contract, uploads \{ticket, sign(ticket)\}, then the smart contract uses the $\mathrm{UAV}_{a}$ 's public key to verify the signature, and then uses the mUAV's public key to verify the signature in the ticket. After confirming their correctness, smart contract writes $\mathrm{H}\left(\mathrm{GrpID}, \mathrm{ID}_{a}\right)$ into the blockchain.

Step (5): mUAV calls the smart contract to search the existence of H(GrpID, ID $a)$.

Step (6): If the search results in step 5 is existing, mUAV will use the public key of $\mathrm{UAV}_{a}$ to send the generated session key SK and timestamp TS2.

$$
\text { mUAV } \stackrel{E_{\mathrm{pa}}\{\text { Ticket, } T S 2\}}{\longrightarrow} U A V_{a}
$$

After that, $\mathrm{UAV}_{a}$ decrypts and checks the timestamp TS2 to obtain the session key SK. So far, $\mathrm{UAV}_{a}$ has successfully joined the group. At this time, all UAVs confirmed to join the group will have the same GrpID and their own confidential IDs, as is shown in the following Figure 3. 


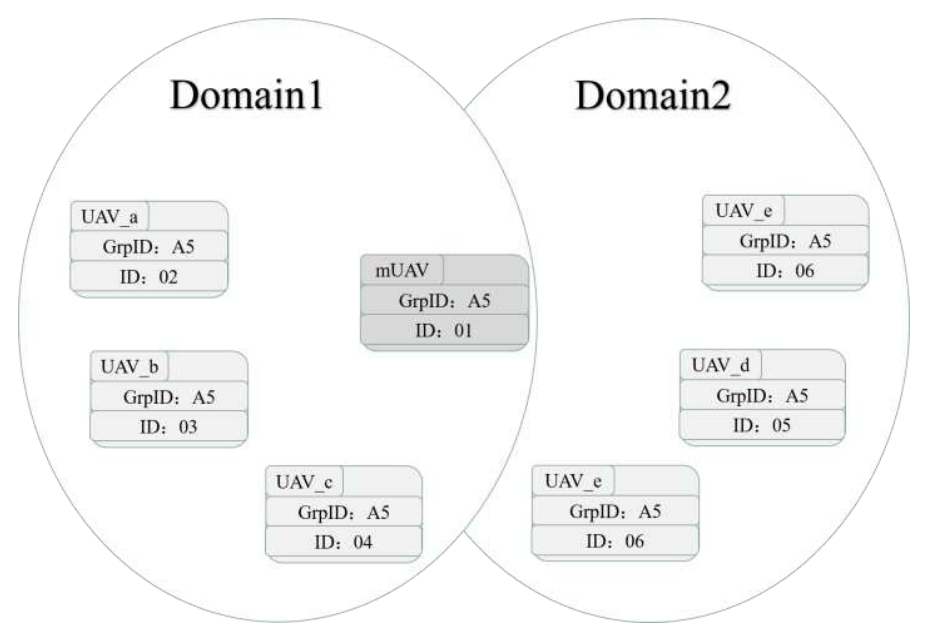

Figure 3 An instance of a group containing UAVs from two domains

\subsection{Identity Information Intra-group Management Method}

During the mission of the UAV group, it will inevitably encounter extreme conditions such as poor or untrustworthy network. In this weak connection environment, it is important to ensure the reliability and efficiency of identity authentication in the group. In this section, a identity information Intra-group management method is proposed. Based on DHT and consistent hash, we have realized offline storage and fast access of UAV identity information.

\section{A. Redundant storage of identity information}

Distributed storage can ensure the lightweight of the UVA, and consistent hash algorithm can ensure the efficiency of resource location. We use the SHA-1 as the hash function.

The ID of Identity information and the UAV's address are mapped to the ring by SHA-1, and authentication information stored in both two successor nodes in a clockwise direction. As shown in Figure 4, O59 resource is stored on the N1 and N8 UVA nodes, and O4 resource is stored on the N8 and N17 UVA nodes. O19 and O25 resources are stored on the N34 and N52 UVA nodes.

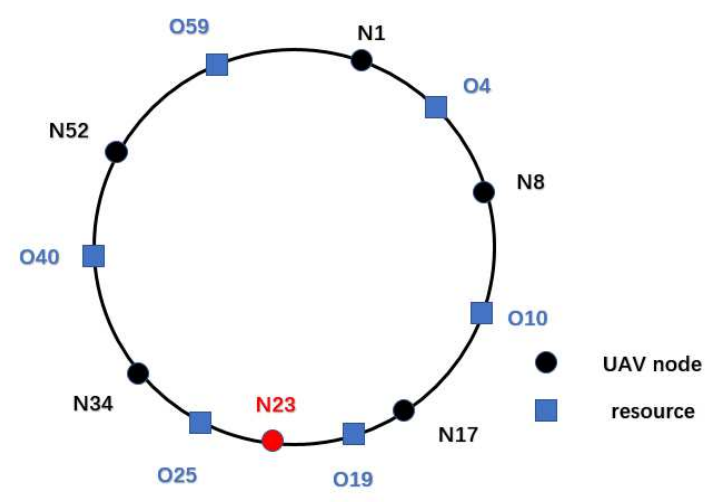

Figure 4 Storage ring of identity information 
From Figure 4, it can be seen that when a new UVA node adds to the group, data migration will only occur between three UVAs according to the clockwise storage rule. For example, when a new UAV node N23 is added to the group, only O19 and O10 resources need to be migrated from N52 and N34 to N23.

\section{B. Rapid location of identity information}

To improve the efficiency of identity information access, each node can quickly access resources by maintaining a Finger table. The i-th item of the table is the resource which hash value is this UVA node plus $2^{(\mathrm{i}-1)}$ and it's location. As shown in Figure 5

(take a hash space of $2^{6}$ as an example).

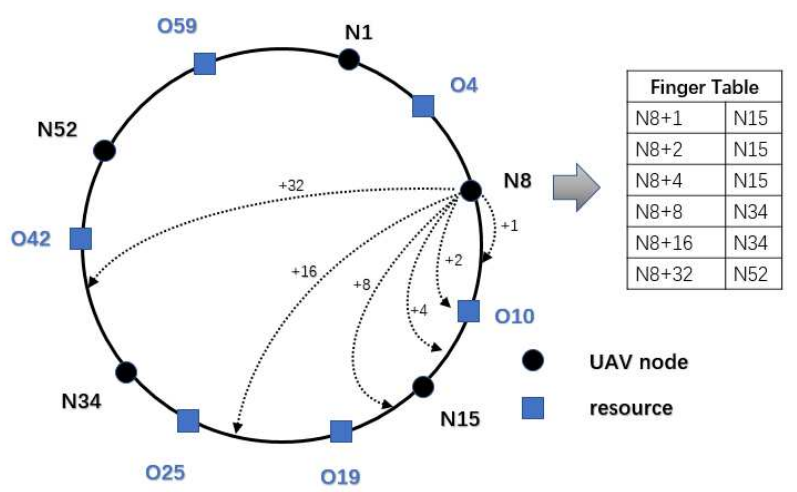

Figure 5 Rapid resource location with finger table

When a UAV node access a resource, it first judges whether its successor node holds the resource. If not, search from the last item in Finger table. When found the hash value of the UVA node is less than the resource node, jump to the UVA node for a new search.

For example, the UVA (N8) needs to access the O42 resource. First look up the own node (N8) and successor node (N15). They do not hold the resource, so the UVA(N8) reverse lookup Finger table. The N34 is the first UVA node with a hash value less than the resource. So jump to the N34 node for a new search. The successor node of the N34 node is N52, which meets the condition of $42 \in(34,52]$, so $\mathrm{O} 42$ resource is located in the N52 UVA nodes. When an uncontrollable factor causes the N52 UAV node to lose connection, the next UAV node N1 can be queried sequentially to read the redundant resources.

\subsection{UAV Authentication in The Group}

During the task process, UAVs will collaborate with each other to complete the mission. The protocol of the UAV authentication process is shown in Figure 6: 


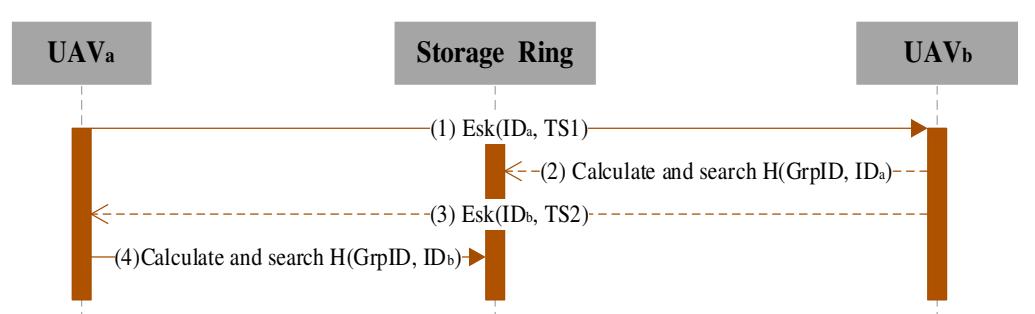

Figure 6 UAV communication

The protocol steps are as follows:

Step (1): $\mathrm{UAV}_{a}$ encrypts its $\mathrm{ID}_{a}$ and timestamp TS1 through the symmetric key sk, and transmits it to $\mathrm{UAV}_{b}$.

$$
\mathrm{UAV}_{a} \stackrel{E_{\mathrm{sk}}\left\{\mathrm{ID}_{a}, T S 1\right\}}{\longrightarrow} \mathrm{UAV}_{b}
$$

Step (2): $\mathrm{UAV}_{b}$ decrypts the message and verifies the timestamp TS1, calculates Hash (GrpID, ID $\mathrm{ID}_{a}$ ), and then uses the Storage Ring to compare and verify the authenticity of $\mathrm{UAV}_{a}$.

Step (3): After $\mathrm{UAV}_{b}$ successfully verifies the identity of $\mathrm{UAV}_{a}$, it encrypts $\left\{\mathrm{ID}_{b}, \mathrm{TS} 2\right\}$ and sends it to $\mathrm{UAV}_{a}$.

$$
\mathrm{UAV}_{b} \stackrel{E_{\mathrm{sk}}\left\{\mathrm{ID}_{b}, \mathrm{TS} 2\right\}}{\longrightarrow} \mathrm{UAV}_{a}
$$

Step (4): After $\mathrm{UAV}_{a}$ decrypts the message, it verifies the timestamp TS2 and calculates Hash $\left(\mathrm{GrpID}, \mathrm{ID}_{b}\right)$, then searches on Storage Ring and verifies the results.

If the result of verification is correct, then enter the communication phase. $\mathrm{UAV}_{a}$ and $\mathrm{UAV}_{b}$ save each other's ID to the local cache, which is convenient for quick verification next time.

\section{Results and Discussion}

In this section, the authentication model will be analyzed from security and computation aspects.

\subsection{Security Analysis}

Our task oriented authentication model for UAVs integrates blockchain technology to improve the flexibility and security. Moreover, a storage ring method is proposed to maintain the identity information, which is used to support the model could be work in weak connection mission environment. A security evaluation of the elaborated proof of concept is given in the following.

When a UAVs mission is initiated, a task-oriented trusted group is established. The authentication between the UAV and the master is executed by calling the blockchain smart contract. The smart contract automatically confirms the authenticity of the identity and writes the confirmation result into the block. All devices can confirm whether the identity authentication is successful by checking the blockchain. On the 
one hand, the blockchain has played the role about an authoritative organization, completing credible and safe identity authentication in a decentralized situation. On the other hand, all IDs of UAVs are stored in the form of hash on the blockchain. In a group, the UAVs also use the ID assigned by the master to authenticate and communicate with each other. If the attacker reads the authentication information stored on the chain by special means, but the attacker can only get the groupID and the related hash value and cannot get UAVs' ID. Finally, each group is accompanied with a task. When the task is over, the group is automatically disbanded. All authenticated devices, tickets, tokens and other identity authentication certificates have all expired. This achieves a certain degree of forward secrecy.

When UAVs group carry out their mission, all UAVs communicated in the trust group, and the communications are kept secret by the session key negotiated when the group building. And the storage ring method is used to maintain identity information while the network is unavailable. First, our model can slove problems such as channel limitation and single point failure that may be caused by a single storage. Second, the session key protects the identity authentication and other communications from being eavesdropped by attackers. In addition, all authentication messages is bound with a timestamp to prevent replay attack. Finally, all authentications information and related authentications operations are stored in the security hardware module (TPM), ensuring that even if the UAV crashes or is captured, it will not affect the identity and communication security of other UAVs.

\subsection{Performance Analysis}

In this subsection, we analyze our ToAM in terms of computation and communication costs by comparing it with BASA[7]. We calculate the computation and communication costs within one task, which includes both group building and communication.

\section{A. Computation overhead}

About computation overhead, the cost will be evaluated through theoretical analysis on most time-consuming operations. This means that complex operations is considered and simple ones is ignored. The simple operations, such as hash operation, integer addition and multiplication cost little time in our computation cost, so they are not considered here. The notations are listed as follows:

- Cs, the cost of performing a symmetric operation (encryption or decryption with a symmetric key).

- Csig, the cost for performing an asymmetric private operation (plaintext decryption or signature using a private key).

- Cver, the cost for performing an asymmetric public operation (plaintext encryption or signature verification using a public key). 
- CECDH, the cost of executing an elliptic curve Diffie-Hellman (ECDH) operation.

- N,the number of UAV joining in the task.

If the ToAM and BASA use the same encryption method and standard, the computation overhead is compared as following Table 2:

Table 2 Computation cost

\begin{tabular}{|l|l|c|c|c|}
\hline \multicolumn{2}{|c|}{ Protocol } & UAV & mUAV & server \\
\hline \multirow{2}{*}{ ToAM } & Group building & $2 * \mathrm{Cs}+\mathrm{Cver}+\mathrm{Csign}$ & $(\mathrm{Cs}+\mathrm{Csign}) * \mathrm{~N}$ & $(\mathrm{Cs}+\mathrm{CECDH}+\mathrm{Cver} * 2) * \mathrm{~N}$ \\
\cline { 2 - 5 } & Group communication & \multicolumn{2}{|c|}{$\mathrm{Cs} 2 * \mathrm{~N}$} & $\backslash$ \\
\hline BSBA & $(2 * \mathrm{Cs}+\mathrm{Cver}+\mathrm{Csign}) * \mathrm{~N}$ & $(2 * \mathrm{Cs}+\mathrm{Cver}+\mathrm{Csign}) * \mathrm{~N}$ \\
\hline
\end{tabular}

As the Table 2 shows, most of the computation cost of ToMA is in the group building, and the computation cost in the group communication is less, which helps to reduce the cost of UAV task execution. In addition, compared with traditional authentication models, such as BSBA, ToMA has less average computation cost and higher verification efficiency, which helps to solve the problem of resource limitations.

\section{B. Communication overhead}

First of all, The length of the information about the task will change with the task. So we only count the length of authentication related messages. First, the master calls the smart contract about 32 bytes and accepts UAV public key about 256 bytes. When a UAV group is created, the master will send a ticket to the UAV, including the the ticket with related signatures and time stamp. Then, UAV sends signature with private key and ticket to the blockchain. After that, the master calls the smart contract to check whether the verification is successful, which is about $32+160$ bytes. Finally, the master sends a 128-byte session key and a 32-byte time stamp to the UAV, which are encrypted with the UAV's public key.

When the group is established, all UAVs need to send the object_ID of 16 bytes to prove their identity, and the receiver sends 160 bytes of hash value to confirm their identity. Because the hash is stored on the storage ring. If we assume there are N UAVs in a group, $\log _{2} N+1$ communications are required for identity verification. It's $\left(\log _{2} N+1\right) * 16$ bytes in total.

Assuming there are N UAVs in a group, The number of all communication bytes is as following Table 3.

Table 3 Communication cost

\begin{tabular}{|c|l|c|}
\hline \multirow{2}{*}{ Protocol link } & \multicolumn{1}{|c|}{ Information category } & Length \\
\hline \multirow{3}{*}{ Group building } & mUAV calls the smart contract and receive the UAV`s public key & $32+256$ \\
\cline { 2 - 3 } & Ticket and time stamp for UAV & $192+32$ \\
\cline { 2 - 3 } & Ticket and related signatures for BS & $192+160$ \\
\hline
\end{tabular}




\begin{tabular}{|c|l|c|}
\hline \multirow{2}{*}{$\begin{array}{c}\text { Group } \\
\text { conversation }\end{array}$} & $\begin{array}{l}\text { mUAV calls the smart contract and receive the related information } \\
\text { the generated session key and time stamp for UAV }\end{array}$ & $32+160$ \\
\cline { 2 - 3 } & Uncertified UAV to verifier & $128+32$ \\
\cline { 2 - 3 } & Verifier find the hash from Storage ring & $\begin{array}{c}\left(\log _{2} N+\right. \\
1) * 16\end{array}$ \\
\hline
\end{tabular}

As the Table 3 shows, communication cost in group building is 1216 bytes, which is less than 1536 bytes of BASA, when both use 32-byte long ID. Secondly, with the storage ring, the communication cost is reduced from $\mathrm{N}^{*} 160+192$ to $\left(\log _{2} N+1\right) *$ 192 in group communication. At the same time, the storage ring also avoids failure of identity authentication by weak connection or single point of failure.

\section{Conclusion}

In this paper, we propose a complete ToAM model, which meets our expectations in terms of security and computation overhead.

From the security analysis in Section 4.1, we can see that our ToAM integrates blockchain technology to improve the flexibility and security. All authentication information is stored in the blockchain in the form of hash, which ensures the security of the group formation and member communication process. In order to ensure the feasibility of working in a weak connection environment, we propose a storage ring method. From the performance evaluation in Section 4.2, most of the computation cost of ToAM is in the group building, and the computation cost in the group communication is less. The average cost of both computation and communication is less than traditional model.

Due to the dynamic nature of UAVs missions and the complexity of the environment, it is difficult for conventional identity authentication models to achieve stable and secure aims. In this paper, a task-oriented authentication model for UAVs based on blockchain is proposed. The whole system is built based on blockchain. Before a task starts, a task-oriented trusted UAVs group is established. All UAVs in the group automatically complete identity authentication through blockchain and smart contracts, and store the authentication information on the blockchain. In the process of task execution, in order to adapt to the weak connection environment, identity information is temporarily stored in the group. And reliability and efficiency are ensured by distributed hash and redundant storage. Lightweight authentication in the group is realized by securely exchanging the identity information of UAVs in the group.

In the future, we will pay more attention to the hierarchical blockchain method and study the lightweight blockchain method suitable for intra-group authentication.

\section{List of Abbreviations}

In our manuscript, we used abbreviations for some words and phrases, here is a list of their meanings. 
1 ToAM: Task-oriented Authentication Model

2 UAV: unmanned aerial vehicles

3 mUAV: master UAV

4 CA: Certificate Authority

5 BAS: Blockchain Authentication Server

6 BS: Business Server

7 TPM: Trusted Platform Module

8 GrpID: Group ID

9 ECDHE: Elliptic Curve Diffie-Hellman Ephemeral

10 ECDLP: Elliptic Curve Discrete Logarithm Problem

11 BASA: Blockchain-Assisted Secure Device Authentication

\section{Declarations}

\subsection{Availability of Data and Material}

Data sharing is not applicable to this article as no new data were created or analyzed in this study.

\subsection{Competing Interests}

The authors declare that they have no competing interests.

\subsection{Funding}

This work was supported by the National Key R\&D Program of China (No. 2018YFC0807500), by National Natural Science Foundation of China (No. U19A2059), and by Ministry of Science and Technology of Sichuan Province Program (No. 2018GZDZX0048,20ZDYF0343).

\subsection{Authors' Contributions}

A.C., K.P., and Z.S. designed the model and conceived the experiments. Z.S. and Z.Y. performed the analysis. K.P., and Z.S. analyzed the data and wrote this paper. G.L. and A.C. helped supervise the project. All authors read and approved the final manuscript.

\subsection{Acknowledgements}

We are thankful to the members of our research team Sijie Zhang, Guanju Li, Deying Liu, Yutong Long and Xincen Zhou, who provided expertise that greatly assisted the research.

\section{References}

1 Hlavacs H (2013) Cooperative enhancement of position accuracy of unmanned aerial vehicles. In: International Conference on Advances in Mobile Computing and Multimedia, pp 326-334.

2 Sharawi MS, Aloi DN, Rawashdeh OA (2010) Design and implementation of embedded printed antenna arrays in small UAV wing structures. IEEE Trans Antennas Propag 
58(8):2531-2538

3 Waharte S., Trigoni N. Supporting search and rescue operations with UAVs. 2010 International Conference on Emerging Security Technologies, IEEE (2010), pp. 142-147.

4 Kennedy, Caroline; Rogers, James I. Virtuous drones. The International Journal of Human Rights . 2015, 19 (2): 211-227.

5 Mungyu Bae, Hwangnam Kim. Authentication and Delegation for Operating a Multi-Drone System, 2019 May 3.

6 Zuobin Xiong, Wei Li, Qilong Han, and Zhipeng Cai. Privacy-Preserving Auto-Driving: a GAN-based Approach to Protect Vehicular Camera Data. 19th IEEE International Conference on Data Mining (ICDM 2019).

7 M. Shen et al., "Blockchain-Assisted Secure Device Authentication for Cross-Domain Industrial IoT," in IEEE Journal on Selected Areas in Communications, vol. 38, no. 5, pp. 942 954, May 2020, doi: 10.1109/JSAC.2020.2980916.

8 Maria L.B.A. Santos, Jéssica C. Carneiro, Antônio M.R. Franco, et al. FLAT: Federated lightweight authentication for the Internet of Things. 2020, 107.

9 Randy Butler, Von Welch, Douglas Engert. A national scale authentication infrastructure, IEEE Computer, vol.33(12), pp: 60- 66, 2000.

10 Jung-San Lee, Chin-Chen Chang, Pen-Yi Chang, et,al. "Anonymous authentication scheme for wireless communications," International Journal of Mobile Communications, vol.16, pp: 590 $-601,2007$.

11 Saide Zhu, Wei Li, Hong Li, Ling Tian, Guangchun Luo, Zhipeng Cai. Coin Hopping Attack in Blockchain-based IoT. IEEE Internet of Things Journal. 6(3): 4614-4626 (2019)

12 Sun, J., Wang, W., Kou, L., Lin, Y., Zhang, L., Da, Q., \& Chen, L. (2017). A data authentication scheme for UAV ad hoc network communication. The Journal of Supercomputing.

13 Chen, Deng, Weng, Chen, Chiu, \& Wu. (2020).A Traceable and Privacy-Preserving Authentication for UAV Communication Control System. Electronics, 9(1), 62

14 Han, W.; Z. An ID-based mutual authentication with key agreement protocol for multiserver environment on elliptic curve cryptosystem. Int. J. Commun. Syst. 2014, 27, 1173-1185.

15 Sarath, G.; Jinwala, D.C.; Patel, S. A Survey on Elliptic Curve Digital Signature Algorithm and its Variants. Electronics 2020, 9, 6230 of 30 Comput. Sci. Inf. Technol. (CSIT)-CSCP 2014, 121-136.

16 Cho, G.; Cho, J.; Hyun, S.; Kim, H. SENTINEL: A Secure and Efficient Authentication Framework for Unmanned Aerial Vehicles. Appl. Sci. 2020, 10, 3149.

17 Wang Hong, Li Jianhua, Lai Chengzhe, et al. A provably secure aggregate authentication scheme for unmanned aerial vehicle cluster networks. 2020, 13(2):53-63

18 Zhipeng Cai, Zhuojun Duan, and Wei Li. Exploiting Multi-Dimensional Task Diversity in Distributed Auctions for Mobile Crowdsensing IEEE Transactions on Mobile Computing. Accepted

19 Allison Gibson, Geethapriya Thamilarasu. Protect Your Pacemaker: Blockchain based Authentication and Consented Authorization for Implanted Medical Devices. 2020, 171:847856.

20 Saide Zhu, Zhipeng Cai, Huafu Hu, Yingshu Li, Wei Li. zkCrowd: A Hybrid Blockchain-based Crowdsourcing Platform. IEEE Transactions on Industrial Informatics (TII). 16(6): 4196-4205 (2020). 


\section{Figure legends}

1 Figure 1 ToAM system model. The ToAM system model includes three layers and several domains. UAV in different domains can dynamically build a drone group with the help of BS.

2 Figure 2 Initialization. This figure shows the process of ToAM formation and initialization.

3 Figure $3 \mathrm{An}$ instance of a group containing UAVs from two domains. This figure shows how UAVs in different domains build groups.

4 Figure 4 Storage ring of identity information. We store the identity information in a Storage ring.

5 Figure 5 Rapid resource location with finger table. Rapid resource location can be seen in this figure.

6 Figure 6 UAV communication. This figure shows the communication plan of UAVs within a group. 
Figures

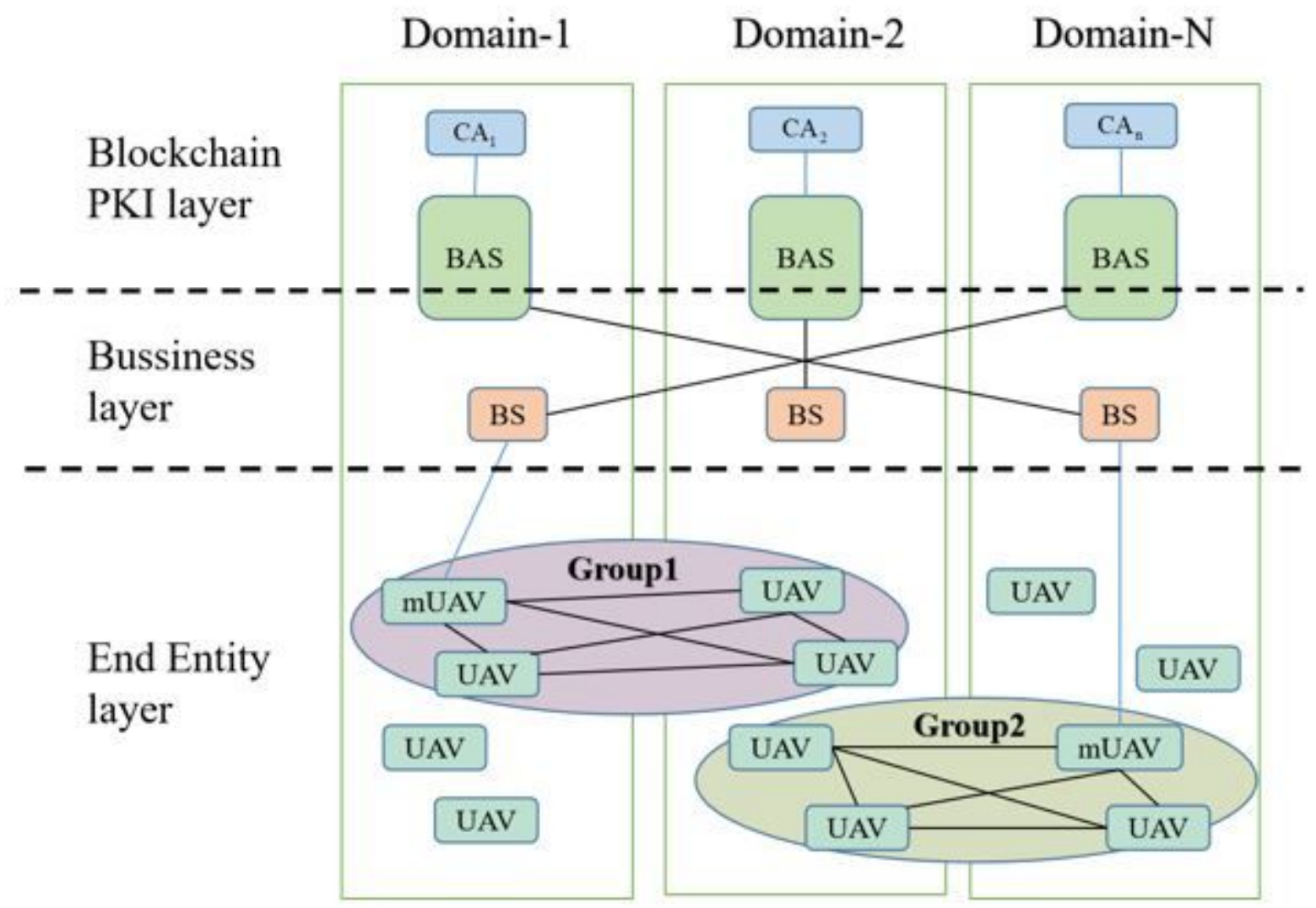

Figure 1

ToAM system model. The ToAM system model includes three layers and several domains. UAV in different domains can dynamically build a drone group with the help of BS. 


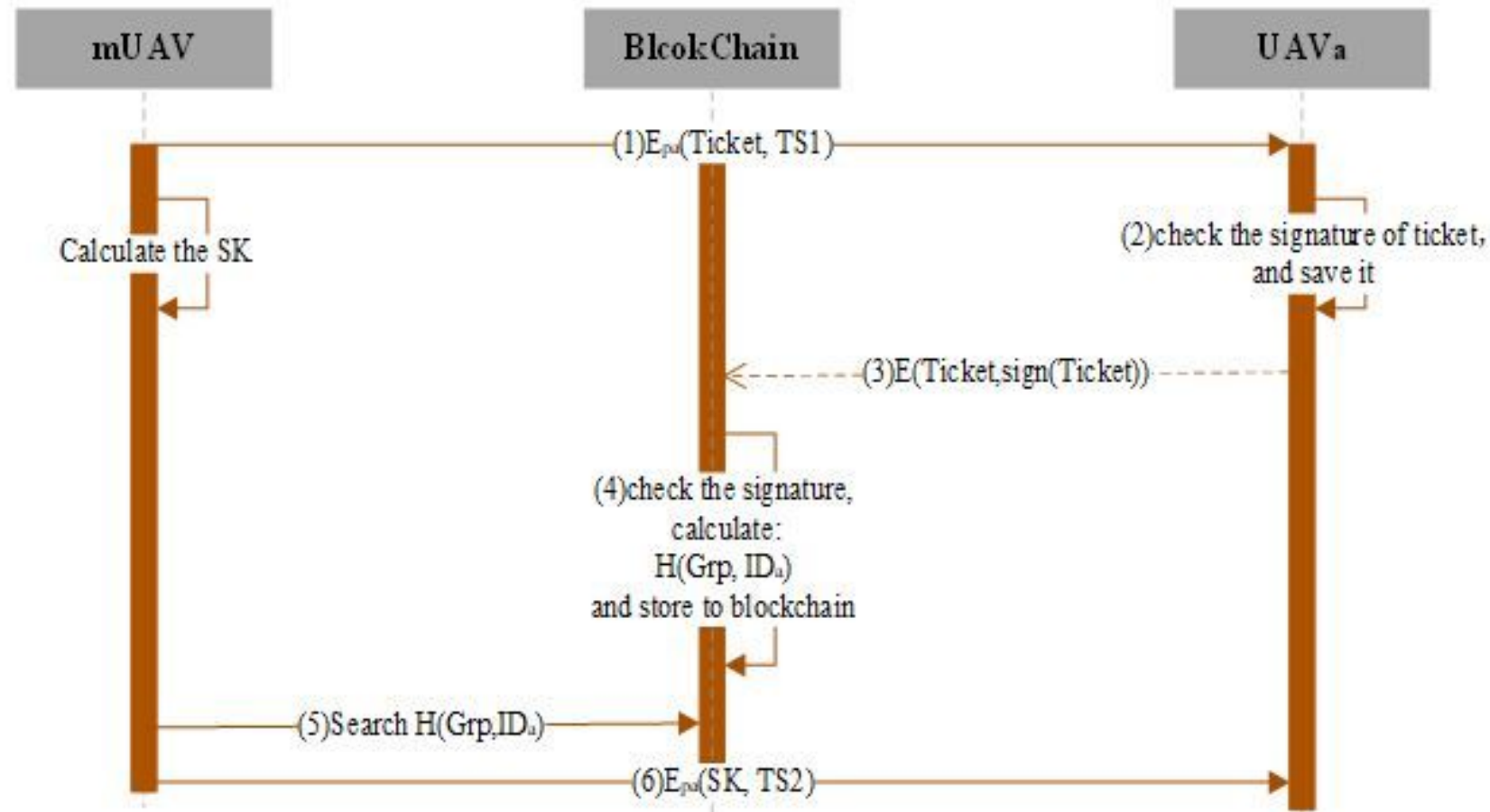

Figure 2

Initialization. This figure shows the process of ToAM formation and initialization.

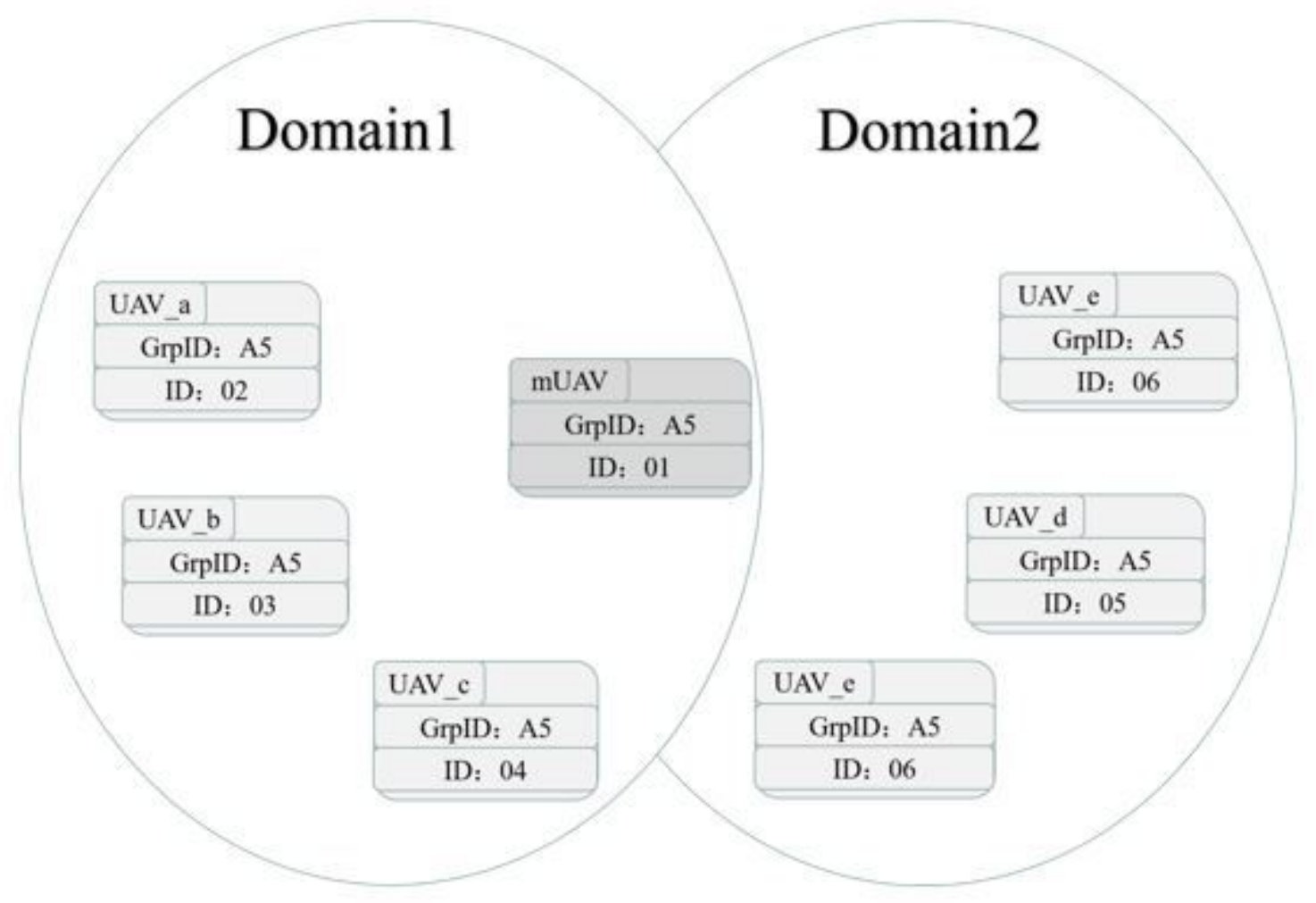

Figure 3 
An instance of a group containing UAVs from two domains. This figure shows how UAVs in different domains build groups.

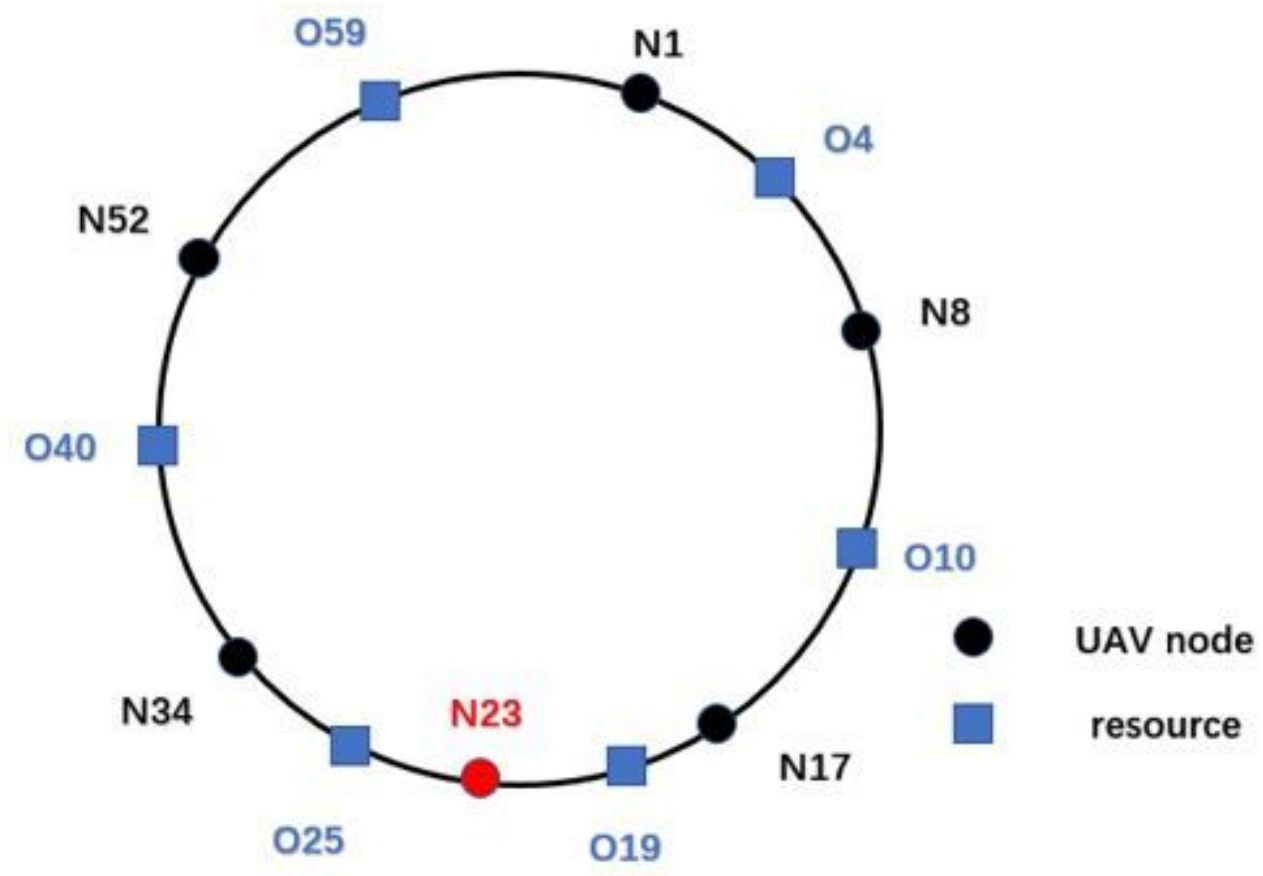

\section{Figure 4}

Storage ring of identity information. We store the identity information in a Storage ring.

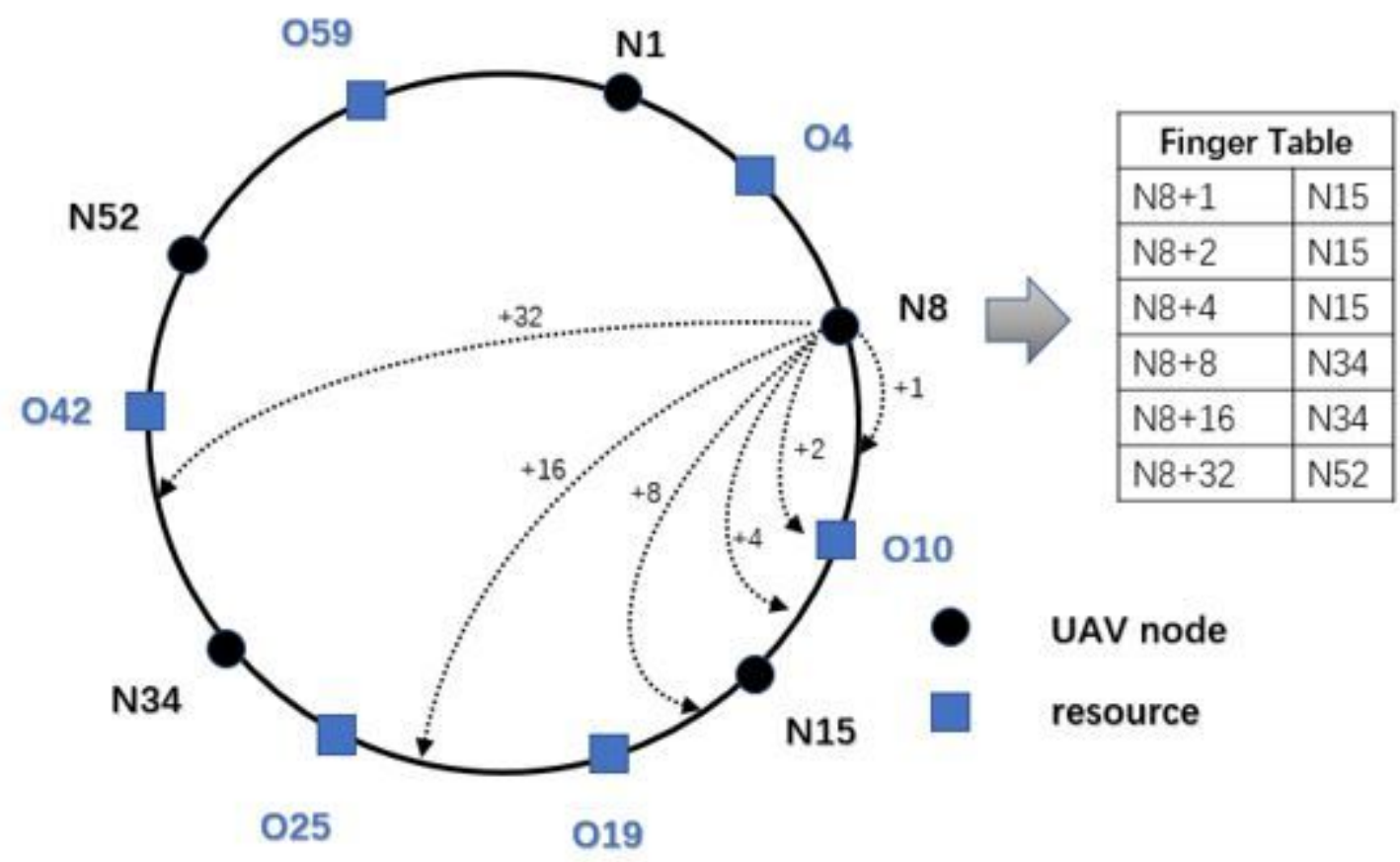


Figure 5

Rapid resource location with finger table. Rapid resource location can be seen in this figure.

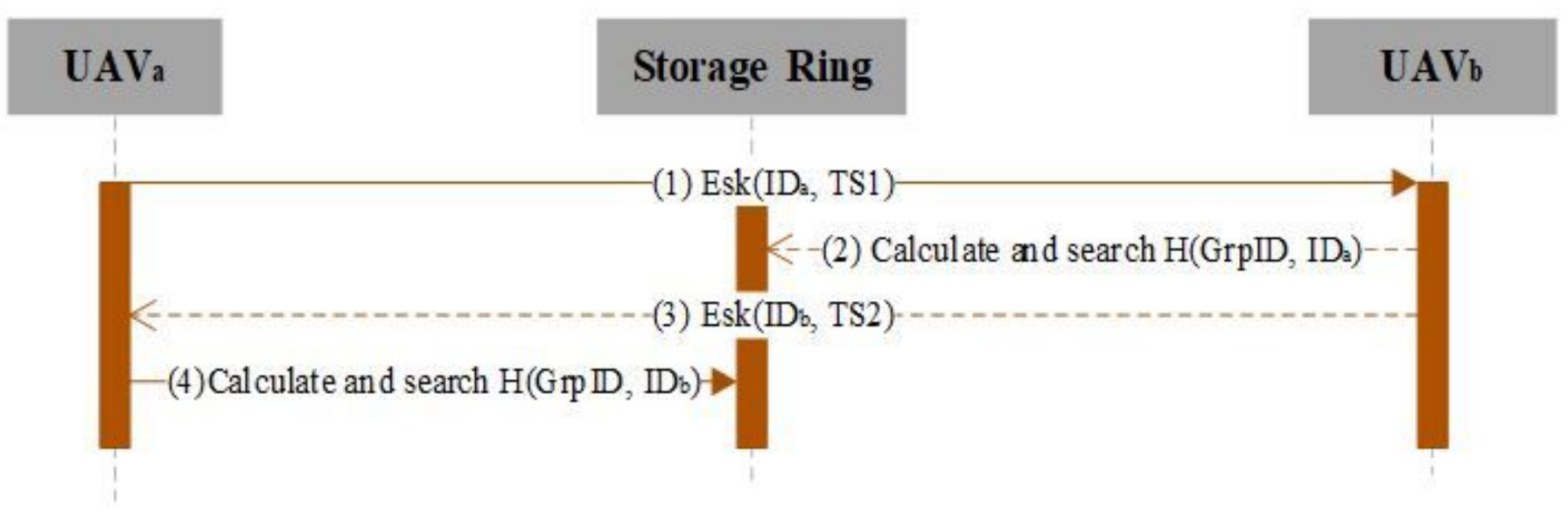

Figure 6

UAV communication. This figure shows the communication plan of UAVs within a group. 